\title{
MDM2 promoter polymorphism del1518 (rs3730485) and its impact on endometrial and ovarian cancer risk
}

Liv B. Gansmo ${ }^{1,2}$, Merete Bjørnslett ${ }^{4,5}$, Mari Kyllesø Halle ${ }^{6,7}$, Helga B. Salvesen ${ }^{6,7^{\wedge}}$, Pål Romundstad ${ }^{3}$, Kristian Hveem $^{3}$, Lars Vatten ${ }^{3}$, Anne Dørum ${ }^{8}$, Per E. Lønning ${ }^{1,2}$ and Stian Knappskog ${ }^{1,2^{*}}$

\begin{abstract}
Background: The del1518 (rs3730485) polymorphism is an in/del variant in the MDM2 promoter P1. The variant is in complete linkage disequilibrium with MDM2 SNP309 (rs2279744) and has previously been found associated with an increased risk of colon cancer. In this study we assessed the impact of MDM2 del1518 on risk of ovarian and endometrial cancer.

Methods: Here, we genotyped del1518 in two large hospital-based series of patients diagnosed with ovarian $(n=1,385)$ or endometrial $(n=1,404)$ cancer and performed risk estimations as compared to the genotype distribution among 1,872 healthy female controls.

Results: In overall analysis we observed no association between del1518 and risk of either ovarian or endometrial cancer. However, stratifying according to SNP309 status, we found the del1518 variant to be associated with a reduced risk of endometrial cancer among individuals carrying the SNP309TT genotype both in the dominant $(\mathrm{OR}=0.64 ; 95 \% \mathrm{Cl}=0.45-0.90)$ and the recessive model $(\mathrm{OR}=0.80 ; 95 \% \mathrm{Cl}=0.65-1.00)$. No such association was observed for ovarian cancer risk.

Conclusion: We found the MDM2 del1518 del variant to be associated with reduced risk of endometrial cancer among individuals carrying the MDM2 SNP309TT genotype.
\end{abstract}

Keywords: MDM2, Del1518, Cancer risk, Ovarian cancer, Endometrial cancer

\section{Background}

The protein product of the Mouse Double Minute 2 homolog (MDM2) gene is one of the main regulators of the tumor suppressor p53. MDM2 inhibits p53, not only by direct binding, but also by directing it to proteasomal degradation. p53, on the other hand, induces MDM2 transcription in response to genotoxic stress [1-5]. Increased levels of MDM2, through mechanisms such as gene amplification, increased transcription and elevated translation has been observed in several human cancers [6-8], and $M D M 2$ overexpression has been suggested to

\footnotetext{
* Correspondence: stian.knappskog@uib.no

'Deceased

${ }^{1}$ Section of Oncology, Department of Clinical Science, University of Bergen, Haukeland University Hospiltal, Jonas Lies veg 87, 5021 Bergen, Norway

${ }^{2}$ Department of Oncology, Haukeland University Hospital, Bergen, Norway

Full list of author information is available at the end of the article
}

be an alternative mechanism of p53 inactivation and tumor promotion $[8,9]$.

Over the last decade, single nucleotide polymorphisms (SNPs) in the MDM2 promoter regions have been reported to affect $M D M 2$ transcription [10-12]. The most studied MDM2 SNP, SNP309T > G (rs2279744) was found to increase the binding affinity between the $M D M 2$ promoter 2 region and the transcription factor Sp1 (specificity protein 1), resulting in increased MDM2 mRNA and protein levels [10]. While initial reports indicated the SNP309G allele to be associated with enhanced risk and early diagnoses of several tumor forms, subsequent data have been at conflict, indicating potential gender as well as ethnic differences [13-20]. A potential reason for these findings may be interaction with other SNP variants located in the $M D M 2$ promoter areas. 
Previously, we and others reported a novel MDM2 SNP located 24 base pairs upstream from SNP309; SNP285G > C (rs117039649) that also affect Sp1 binding to the $M D M 2$ promoter [11, 21, 22]. Notably, this SNP was found to be differentially distributed across different ethnic groups [11, 23].

Contrasting SNP309 and SNP285, which both are located in the MDM2 promoter P2, the 40 bps insertion/ deletion polymorphism del1518 (rs3730485) is located in the $M D M 2$ promoter P1. The del1518 variant has been reported to be in linkage disequilibrium (LD) with SNP309, forming a SNP309T/del1518 del haplotype $[24,25]$. While the del variant has been associated with decreased $M D M 2$ expression [12], results so far have linked the del1518 del allele to an increased risk of hepatocellular carcinoma, colon cancer and uterine leiomyoma [25-27] but with no associations to epithelial ovarian cancer, esophageal squamous cell carcinoma, breast, lung or prostate cancer risk [24, 25, 28-30].

In the present study we assessed the potential association between del1518 and risk for ovarian and endometrial cancer in large hospital based sample sets, previously genotyped for SNP309 and SNP285, enabling assessment of potential synergisms between the three SNPs.

\section{Methods}

\section{Study populations}

All cases included in this case-control study were obtained from hospital-based cohorts of Norwegian patients of whom the great majority were Caucasians diagnosed with endometrial $(n=1,404)$ and ovarian cancer $(n=1,385)$ described in detail previously [31]. For comparison, we used the previously reported genotypes of the female fraction $(n=1,872)$ from a sample set of 3,749 healthy Norwegian individuals [32]. These individuals were initially drawn from the population based Cohort of Norway (CONOR) study [33].

For the endometrial cancer samples we had histological status for 1,320 and for the ovarian cancer samples histopathological status was available for 1,071 [31].

\section{MDM2 genotyping}

All samples were genotyped for $M D M 2$ del1518 by using DNA extracted from white blood cells as previously described [25]. Briefly, the region of MDM2 containing the del1518 indel polymorphism was amplified by PCR, and the insertion and deletion alleles were separated and visualized by electrophoresis in a 3\% agarose gel pre-stained with GelRed ${ }^{\text {tm }}$ Nucleic Acid Gel Stain (BIOTIUM).

MDM2 SNP309 genotypes were extracted from previously published data from the same individuals [11, 21, 25, 31, 32].

\section{Statistical analysis}

Since we, and others, previously have shown the $M D M 2$ del1518 to be in strong linkage disequilibrium with the MDM2 SNP309 (the del1518 del-allele being linked to the SNP309T-allele) [24, 25], we followed an analysis plan specified up-front of statistical assessments. First we performed overall risk assessments including all EC and $\mathrm{OC}$ patients. Then we performed sub-analyses, stratifying the data according to histology and according to SNP309-status before assessing putative associations between del1518 and cancer risk.

Possible associations between del1518 and cancer risk, both in total and in stratified groups, were evaluated by Odds Ratios (OR) and Fisher's exact tests. ORs are given with 95\% confidence intervals (CI), and $p$-values are given as two-sided. $P$-values from Fisher's exact tests are given as two-sided and cumulative.

All statistical analyses were performed using the IBM SPSS statistics (version 22) software package.

\section{Results \\ Distribution of del1518}

The genotype distribution of the del1518 ins/del variant among the healthy controls was found to be in Hardy-Weinberg equilibrium $(p>0.8)$, with a minor allele frequency (MAF) of 0.43 (genotypes: del1518 ins $/$ ins $=34.0 \%$; del1518 ins $/ \mathrm{del}=46.9 \%$ and del1519 $\mathrm{del} / \mathrm{del}=19.2 \%$ ).

An overview of the del1518 genotype distribution in the healthy controls and the two cancer types are summarized in Table 1. Among the healthy controls, the del1518 del allele was observed in individuals carrying the SNP309TT and 309TG genotypes only. Amongst the patients we found one ovarian cancer patient harboring the del1518 del/del - SNP309TG genotype and two patients (one diagnosed with endometrial- and one diagnosed with ovarian cancer) harboring the del1518 ins/del - SNP309GG genotype. Thus, del1518 was found to be in strong linkage disequilibrium (LD) with SNP309 $\left(D^{\prime}=0.997\right.$ for endometrial cancer cases and $D^{\prime}=0.994$ for ovarian cancer cases) across all groups of individuals. Given that the SNP285C-variant is linked to the SNP309G-allele, we here, in line with previous observations [25], found no evidence of alleles carrying both SNP285C and del1518 del.

\section{MDM2 del1518 and risk of endometrial - and ovarian cancer}

In order to evaluate the potential impact of $M D M 2$ del1518 status on endometrial and ovarian cancer risk, we evaluated the OR by comparing the frequency of the MDM2 del1518 genotypes observed in ovarian and endometrial cancer cases to the SNP status among the 1,872 healthy female controls. No significant association 
Table 1 MDM2 del1518 distribution and cancer risk (OR)

\begin{tabular}{|c|c|c|c|c|c|c|c|}
\hline \multirow[t]{2}{*}{ Cases/controls } & \multicolumn{3}{|c|}{ Genotype del1518 n (\%) } & \multirow{2}{*}{$\begin{array}{l}\text { OR }(95 \% \text { CI) del1518 } \\
\text { Dominant model }^{\mathrm{a}}\end{array}$} & \multirow[t]{2}{*}{ Fisher exact } & \multirow{2}{*}{$\begin{array}{l}\text { OR }(95 \% \text { Cl) del1518 } \\
\text { Recessive model }^{b}\end{array}$} & \multirow[t]{2}{*}{ Fisher exac } \\
\hline & ins/ins & ins/del & del/del & & & & \\
\hline Healthy Controls & $636(34.0)$ & $877(46.9)$ & $359(19.2)$ & 1.00 & - & 1.00 & - \\
\hline Ovarian cancer & $484(35.0)$ & $655(47.3)$ & $246(17.7)$ & $0.96(0.83-1.11)$ & 0.576 & $0.91(0.76-1.09)$ & 0.316 \\
\hline Endometrial cancer & $492(35.0)$ & $664(47.3)$ & $248(17.7)$ & $0.95(0.83-1.10)$ & 0.528 & $0.90(0.76-1.08)$ & 0.276 \\
\hline
\end{tabular}

${ }^{a} \mathrm{del} / \mathrm{del}+$ ins/del versus ins/ins

${ }^{b} \mathrm{del} / \mathrm{del}$ versus ins/del + ins/ins

between MDM2 del1518 and risk of either of the two cancer types was found whether applying the dominant or the recessive model (Table 1; Fig. 1a and b).

Next, we stratified the endometrial malignant lesions and ovarian cancers into histological subgroups (endometrioid, adenosquamous, clear cell, serous papillary, hyperplasia, carcinosarcoma and undifferentiated/ other types endometrial cancer; high-grade serous ovarian cancer [HGSOC], low-grade ovarian cancer [LGSOC], clear cell ovarian cancer, endometrioid ovarian cancer and mucinous ovarian cancer). No associations between MDM2 del1518 ins/del distribution and cancer risk were observed in either of these subgroups (Additional file 1: Tables S1 and Additional file 2: Table S2).

\section{Impact of MDM2 del1518 status within SNP309 genotype subgroups}

Given that the MDM2 del1518 del allele and the SNP309T was found to be in strong LD forming a distinct del1518del/SNP309T haplotype, we performed refined analyses, restricting our OR estimates to individuals carrying the SNP309TT or SNP309TG genotypes. Within the subgroup of individuals carrying the SNP309TT genotype, we observed an association between the del1518 del allele and a reduced risk of endometrial cancer, both when applying the dominant $(\mathrm{OR}=0.64, \mathrm{CI}=0.45-0.90)$ and the recessive model $(\mathrm{OR}=0.80, \mathrm{CI}=0.65-1.00$; Table 2; Fig. 1a and b). In contrast, no significant association was observed among the ovarian cancer patients. Among individuals harboring the SNP309TG genotype, no association to cancer risk was recorded in either of the two cancer forms (Table 3).

The majority of the endometrial cancer patients had tumors of endometroid histology. Within this subgroup (endometroid histology and SNP309TT-genotype), we found the del1518 del allele to be associated with reduced cancer risk, similar to the reduction observed for the total EC-sample set $(\mathrm{OR}=0.64, \mathrm{CI}=0.44-0.92$; dominant model; Additional file 3: Table S3). Regarding the other histology subgroups, these were too small for formal statistical risk analysis among individuals harboring the
A

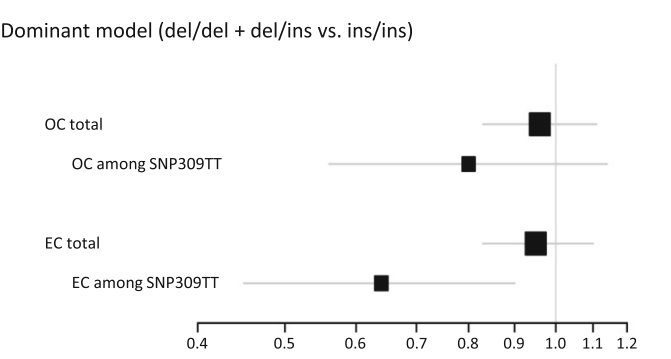

B

Recessive model (del/del vs. del/ins + ins/ins)

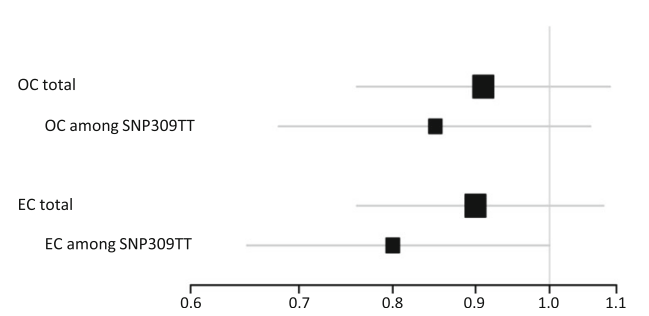

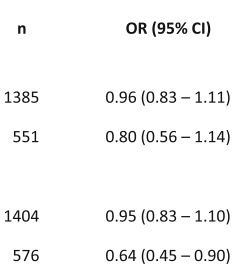

n $\quad$ OR $(95 \% \mathrm{Cl})$

$1385 \quad 0.91(0.76-1.09)$

$551 \quad 0.85(0.68-1.06)$

$1404 \quad 0.90(0.76-1.08)$

$576 \quad 0.80(0.65-1.00)$

Fig. 1 MDM2 del1518 and impact on risk of ovarian and endometrial cancer. Forest plots showing the impact of MDM2 del1518, in the total sample sets, and within the subgroup of SNP309, on ovarian (OC) and endometrial (EC) cancer risk. a dominant model and $\mathbf{b}$ recessive model 
Table 2 MDM2 del1518 among SNP309TT

\begin{tabular}{|c|c|c|c|c|c|c|c|}
\hline \multirow[t]{2}{*}{ Cases/controls } & \multicolumn{3}{|c|}{ Genotype del1518 n (\%) } & \multirow{2}{*}{$\begin{array}{l}\text { OR }(95 \% \mathrm{Cl}) \text { del1518 } \\
\text { Dominant model }\end{array}$} & \multirow[t]{2}{*}{ Fisher exact } & \multirow{2}{*}{$\begin{array}{l}\text { OR }(95 \% \mathrm{Cl}) \text { del1518 } \\
\text { Recessive model }^{\mathrm{b}}\end{array}$} & \multirow[t]{2}{*}{ Fisher exac } \\
\hline & ins/ins & ins/del & del/del & & & & \\
\hline Healthy Controls & $70(9.5)$ & $311(42.0)$ & $359(48.5)$ & 1.00 & - & 1.00 & \\
\hline Ovarian cancer & $64(11.6)$ & $242(44.0)$ & $245(44.5)$ & $0.80(0.56-1.14)$ & 0.231 & $0.85(0.68-1.06)$ & 0.159 \\
\hline Endometrial cancer & $81(14.1)$ & $247(42.9)$ & $248(43.1)$ & $0.64(0.45-0.90)$ & 0.011 & $0.80(0.65-1.00)$ & 0.051 \\
\hline
\end{tabular}

${ }^{a} \mathrm{del} / \mathrm{del}+$ ins/del versus ins/ins

${ }^{\mathrm{b}} \mathrm{del} / \mathrm{del}$ versus ins/del + ins/ins

SNP309TT genotype (pooled data for these subgroups shown in Additional file 3: Table S3).

\section{Discussion}

Previous studies have revealed MDM2 promoter P2 SNP variants like SNP309T > G and SNP285G > C to modulate gene transcription and affect cancer risk $[10,11]$.

In the present study, we explored the impact of a third MDM2 polymorphism, del1518 ins/del, located in promoter P1, on the risk of endometrial and ovarian cancer in a Caucasian population. Similar to what has been previously reported in Han Chinese [24], we confirmed del1518del/ins to be in strong linkage disequilibrium with the SNP309, forming a distinct MDM2 del1518del/ SNP309T haplotype [24, 25] with a MAF different from Chinese $(\mathrm{MAF}=0.30)$ [24].

We did not detect an effect of del1518 status on the risk of endometrial or ovarian cancer in the general population. Howevver, based on previous findings indicating different $M D M 2$ promoter SNPs may act in concert [11] we performed subgroup analyses stratifying individuals with respect to SNP309 genotypes. (Linkage disequilibrium precluded assessment of SNP285 subgroups since all SNP285C-alleles are linked to SNP309G-alleles while del1518 del locates to the SNP309T-allele [23]).

Doing so, we found the del1518 del- allele to be associated with a reduced risk of endometrial but not for ovarian cancer applying a dominant as well as recessive model among individuals harboring the SNP309TT genotype (risk reduction of $36 \%$ and $20 \%$, respectively). This finding is in line with previous in vitro luciferase gene reporter assay findings, showing the presence of the del1518 del allele to result in complete abrogation of promoter activity [12]. Lack of effect on ovarian cancer risk is also in accordance with the results from a previous study performed in Chinese individuals [28].
However, other studies have found the del1518 del allele to confer an increased risk for other cancer forms like hepatocellular carcinomas [26] and left sided colon cancer among individuals carrying the SNP309TG genotype [25], as well as increased risk for uterine leiomyomas (non-cancerous growths in the uterus) [27], but no associations to epithelial ovarian cancer, esophageal squamous cell carcinoma, breast, lung or prostate cancer risk [24, 25, 28-30]. Taken together, these findings suggests the del 1518 ins/del variant may have different effects on risk for cancer development in different organs, partly dependent on interactions with other MDM2 SNP variants, similar to what has been observed for promoter variants like SNP309 and SNP285 [11, 17, $21,32]$. Interestingly, both endometrial and ovarian cancer risk has previously been found to be reduced by the SNP285C-allele [11, 21]. Further, the SNP309G-allele has been found to be associated with an increased but also a reduced cancer risk across different tissues [16, 18, 34, 35]. Also the fact that mice carrying the human $M D M 2$ SNP309G allele only displayed elevated MDM2 expression in a few tissues [36] supports the hypothesis that SNP309 may act as a cancer risk factor in distinct tissues only. Thus, our observation of del1518 del variant reducing the risk of endometrial but not ovarian cancer may indicate that this variant is modulating the binding of transcription factors that are differentially expressed in these two tissues.

Given that we previously have found a SNP in the MDM4 3 'UTR (SNP34091) to be associated with increased risk of serous, and in particular high grade serous ovarian cancer [31], we performed subgroup analysis based on histology status with respect to del1581. These subgroup analyses did not reveal any histology specific effects of del1518 status, indicating that this variant have no effect on tumor progression.

Table 3 MDM2 del1518 among SNP309TG

\begin{tabular}{|c|c|c|c|c|c|}
\hline \multirow[t]{2}{*}{ Cases/controls } & \multicolumn{3}{|c|}{ Genotype del1518 n (\%) } & \multirow{2}{*}{$\begin{array}{l}\text { OR }(95 \% \text { Cl) del1518 } \\
\text { Ins/del vs. ins/ins }\end{array}$} & \multirow[t]{2}{*}{ Fisher exact } \\
\hline & ins/ins & ins/del & $\mathrm{del} / \mathrm{del}^{\mathrm{a}}$ & & \\
\hline Healthy Controls & $312(35.5)$ & $566(64.5)$ & - & 1.00 & - \\
\hline Ovarian cancer & $213(34.1)$ & $412(65.9)$ & - & $1.07(0.86-1.32)$ & 0.583 \\
\hline Endometrial cancer & $226(35.2)$ & $416(64.8)$ & - & $1.02(0.82-1.26)$ & 0.914 \\
\hline
\end{tabular}

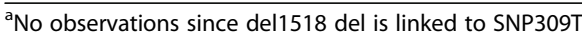




\section{Conclusion}

In conclusion we find the MDM2 SNP del1518 del variant to be associated with reduced risk of endometrial cancer among individuals carrying the SNP309TT genotype, an observation warranting confirmation in independent studies.

\section{Additional files}

Additional file 1: Table S1. MDM2 del158 genotype distribution in OC subgroups. (DOCX $17 \mathrm{~kb}$ )

Additional file 2: Table S2. MDM2 del158 genotype distribution in EC subgroups. (DOCX $17 \mathrm{~kb}$ )

Additional file 3: Table S3. MDM2 del158 genotype distribution among individuals with SNP309TT genitype in EC subgroups. (DOCX $15 \mathrm{~kb}$ )

Additional file 4: Table S4. Raw data; genotypes for all individuals in study. (XLSX $118 \mathrm{~kb}$ )

\section{Abbreviations}

Cl: Confidence intervals; CONOR: Cohort of Norway; EC: Endometrial cancer: HGSOC: High grade serous ovarian cancer; LD: Linkage disequilibrium; LGSOC: Low grade serous ovarian cancer; MDM2: Mouse Double Minute 2; OC: Ovarian cancer; OR: Odds Ratios; SNP: Single nucleotide polymorphism; Sp1: Specificity protein 1

\section{Acknowledgements}

We thank Silje Bjørnklett and Christine Eriksen for technical assistance.

\section{Funding}

This study was supported by grants from the Norwegian Cancer Society, the Norwegian Health Region West funding body, the Norwegian Research Council and the Bergen Research Foundation. None of the funding sources had any role in the study design, data collection/analyses, interpretation of data, or writing of the manuscript.

\section{Availability of data and materials}

The data sets supporting the results of this article are included within the article and its supplementary files (raw data is provided in Additional file 4: Table S4).

\section{Authors' contributions}

PEL and SK designed the study. LBG did all the genotyping and statistical analysis. LBG, SK and PEL interpret the data and wrote the paper. MB, $M K H, H B S, P R, K H, L V$ and $A D$ provided patient samples and clinical data, and reviewed the manuscript. All authors read and approved the final manuscript.

\section{Competing interests}

The authors declare that they have no competing interests.

\section{Consent for publication}

Not applicable.

\section{Ethics approval and consent to participate}

All patients and sample donors enrolled have given written informed consent to the use of their biomaterial for scientific purposes, and the study was approved by the Regional Committee for Medical and Health Research Ethics in - Central Norway (REK Midt-Norge).

\section{Author details}

'Section of Oncology, Department of Clinical Science, University of Bergen, Haukeland University Hospiltal, Jonas Lies veg 87, 5021 Bergen, Norway. ${ }^{2}$ Department of Oncology, Haukeland University Hospital, Bergen, Norway. ${ }^{3}$ Department of Public Health, Faculty of Medicine, Norwegian University of Science and Technology, Trondheim, Norway. ${ }^{4}$ Department of Molecular Oncology, Oslo University Hospital Radium Hospitalet, Oslo, Norway. ${ }^{5}$ Institute for Cancer Research, University of Oslo, Oslo, Norway. ${ }^{6}$ Department of Gynecology and Obstetrics, Haukeland University Hospital, Bergen, Norway. ${ }^{7}$ Centre for Cancer Biomarkers (CCBIO), Department of Clinical Science, University of Bergen, Bergen, Norway. ${ }^{8}$ Department of Gynecologic Oncology, Oslo University Hospital, Norwegian Radium Hospital, Oslo, Norway

Received: 12 October 2016 Accepted: 28 January 2017

Published online: 03 February 2017

\section{References}

1. Haupt Y, Maya R, Kazaz A, Oren M. Mdm2 promotes the rapid degradation of p53. Nature. 1997;387:296-9.

2. Honda R, Tanaka H, Yasuda H. Oncoprotein MDM2 is a ubiquitin ligase E3 for tumor suppressor p53. FEBS Lett. 1997;420:25-7.

3. Kubbutat $\mathrm{MHG}$, Jones $\mathrm{SN}$, Vousden $\mathrm{KH}$. Regulation of $\mathrm{p} 53$ stability by Mdm2. Nature. 1997;387:299-303.

4. Momand J, Zambetti GP, Olson DC, George D, Levine AJ. The mdm-2 oncogene product forms a complex with the p53 protein and inhibits p53-mediated transactivation. Cell. 1992;69:1237-45.

5. Oliner JD, Pietenpol JA, Thiagalingam S, Gyuris J, Kinzler KW, Vogelstein B. Oncoprotein MDM2 conceals the activation domain of tumour suppressor p53. Nature. 1993:362:857-60.

6. Landers JE, Cassel SL, George DL. Translational enhancement of mdm2 oncogene expression in human tumor cells containing a stabilized wild-type p53 protein. Cancer Res. 1997;57:3562-8.

7. Momand J, Jung D, Wilczynski S, Niland J. The MDM2 gene amplification database. Nucleic Acids Res. 1998;26:3453-9.

8. Oliner JD, Kinzler KW, Meltzer PS, George DL, Vogelstein B. Amplification of a gene encoding a p53-associated protein in human sarcomas. Nature. 1992:358:80-3.

9. Bartel F, Meye A, Wurl P, Kappler M, Bache M, Lautenschlager C, et al. Amplification of the MDM2 gene, but not expression of splice variants of MDM2 MRNA, is associated with prognosis in soft tissue sarcoma. Int J Cancer. 2001;95:168-75.

10. Bond GL, Hu W, Bond EE, Robins H, Lutzker SG, Arva NC, et al. A single nucleotide polymorphism in the MDM2 promoter attenuates the p53 tumor suppressor pathway and accelerates tumor formation in humans. Cell. 2004;119:591-602

11. Knappskog S, Bjornslett M, Myklebust LM, Huijts PE, Vreeswijk MP, Edvardsen H, et al. The MDM2 promoter SNP285C/309G haplotype diminishes Sp1 transcription factor binding and reduces risk for breast and ovarian cancer in caucasians. Cancer Cell. 2011;19:273-82.

12. Lalonde ME, Ouimet M, Lariviere M, Kritikou EA, Sinnett D. Identification of functional DNA variants in the constitutive promoter region of MDM2. Hum Genomics. 2012;6:15.

13. Bai J, Dai J, Yu H, Shen H, Chen F. Cigarette smoking, MDM2 SNP309, gene-environment interactions, and lung cancer risk: a meta-analysis. J Toxicol Environ Health A. 2009:72:677-82.

14. Economopoulos KP, Sergentanis TN. Differential effects of MDM2 SNP309 polymorphism on breast cancer risk along with race: a meta-analysis. Breast Cancer Res Treat. 2010;120:211-6.

15. Hu Z, Jin G, Wang L, Chen F, Wang X, Shen H. MDM2 promoter polymorphism SNP309 contributes to tumor susceptibility: evidence from 21 case-control studies. Cancer Epidemiol Biomarkers Prev. 2007;16:2717-23.

16. Liu G, Jiang D, Shen S, Yu L. Murine double minute 2 promoter SNP309 polymorphism and prostate cancer risk: a meta-analysis. Int J Urol. 2012;19:914-20.

17. Wan Y, Wu W, Yin Z, Guan P, Zhou B. MDM2 SNP309, gene-gene interaction, and tumor susceptibility: an updated meta-analysis. BMC Cancer. 2011;11:208

18. Wilkening S, Bermejo JL, Hemminki K. MDM2 SNP309 and cancer risk: a combined analysis. Carcinogenesis. 2007;28:2262-7.

19. Zhao E, Cui D, Yuan L, Lu W. MDM2 SNP309 polymorphism and breast cancer risk: a meta-analysis. Mol Biol Rep. 2012;39:3471-7.

20. Gui XH, Qiu LX, Zhang HF, Zhang DP, Zhong WZ, Li J, et al. MDM2 $309 \mathrm{~T} / \mathrm{G}$ polymorphism is associated with lung cancer risk among asians. Eur J Cancer. 2009;45:2023-6.

21. Knappskog S, Trovik J, Marcickiewicz J, Tingulstad S, Staff AC, et al. MoMaTEC study group. SNP285C modulates oestrogen receptor/Sp1 binding to the MDM2 promoter and reduces the risk of endometrial but not prostatic cancer. Eur J Cancer. 2012;48:1988-96. 
22. Paulin F, O'Neill M, McGregor G, Cassidy A, Ashfield A, Ali C, et al. MDM2 SNP309 is associated with high grade node positive breast tumours and is in linkage disequilibrium with a novel MDM2 intron 1 polymorphism. BMC Cancer. 2008;8:281

23. Knappskog S, Gansmo LB, Dibirova K, Metspalu A, Cybulski C, Peterlongo P, et al. Population distribution and ancestry of the cancer protective MDM2 SNP285 (rs117039649). Oncotarget. 2014;5:8223-34.

24. Hu Z, Ma H, Lu D, Qian J, Zhou J, Chen Y, et al. Genetic variants in the MDM2 promoter and lung cancer risk in a chinese population. Int J Cancer. 2006;118:1275-8.

25. Gansmo LB, Vatten L, Romundstad P, Hveem K, Ryan BM, Harris CC, et al. Associations between the MDM2 promoter P1 polymorphism del1518 (rs3730485) and incidence of cancer of the breast, lung, colon and prostate, Oncotarget. 2016;7:28637-46.

26. Dong D, Gao X, Zhu Z, Yu Q, Bian S, Gao Y. A 40-bp insertion/deletion polymorphism in the constitutive promoter of MDM2 confers risk for hepatocellular carcinoma in a chinese population. Gene. 2012;497:66-70.

27. Salimi S, Hajizadeh A, Khodamian M, Pejman A, Fazeli K, Yaghmaei M. Age-dependent association of MDM2 promoter polymorphisms and uterine leiomyoma in south-east iran: a preliminary report. J Obstet Gynaecol Res. 2015:41:729-34.

28. Kang S, Wang DJ, Li WS, Wang N, Zhou RM, Sun DL, et al. Association of p73 and MDM2 polymorphisms with the risk of epithelial ovarian cancer in chinese women. Int J Gynecol Cancer. 2009;19:572-7.

29. Ma H, Hu Z, Zhai X, Wang S, Wang X, Qin J, et al. Polymorphisms in the MDM2 promoter and risk of breast cancer: a case-control analysis in a chinese population. Cancer Lett. 2006;240:261-7.

30. Ma J, Zhang J, Ning T, Chen Z, Xu C. Association of genetic polymorphisms in MDM2, PTEN and P53 with risk of esophageal squamous cell carcinoma. J Hum Genet. 2012;57:261-4.

31. Gansmo LB, Bjørnslett M, Halle MK, Salvesen HB, Dørum A, Birkeland E, et al. The MDM4 SNP34091 (rs4245739) C-allele is associated with increased risk of ovarian-but not endometrial cancer. Tumor Biol. 2016:37:10697-702.

32. Gansmo LB, Knappskog S, Romundstad P, Hveem K, Vatten L, Lonning PE. Influence of MDM2 SNP309 and SNP285 status on the risk of cancer in the breast, prostate, lung and colon. Int J Cancer. 2015;137:96-103.

33. Naess O, Sogaard AJ, Arnesen E, Beckstrom AC, Bjertness E, Engeland A, et al. Cohort profile: cohort of norway (CONOR). Int J Epidemiol. 2008:37:481-5.

34. Xie L, Sun Y, Chen T, Tian D, Li Y, Zhang Y, et al. Association between MDM2 SNP309 T > G polymorphism and the risk of bladder cancer: new data in a chinese population and an updated meta-analysis. OncoTargets Ther. 2015:8:3679-90.

35. Bond GL, Hirshfield KM, Kirchhoff T, Alexe G, Bond EE, Robins H, et al. MDM2 SNP309 accelerates tumor formation in a gender-specific and hormone-dependent manner. Cancer Res. 2006;66:5104-10.

36. Post SM, Quintas-Cardama A, Pant V, Iwakuma T, Hamir A, Jackson JG, et al. A high-frequency regulatory polymorphism in the p53 pathway accelerates tumor development. Cancer Cell. 2010;18:220-30.

\section{Submit your next manuscript to BioMed Central and we will help you at every step:}

- We accept pre-submission inquiries

- Our selector tool helps you to find the most relevant journal

- We provide round the clock customer support

- Convenient online submission

- Thorough peer review

- Inclusion in PubMed and all major indexing services

- Maximum visibility for your research

Submit your manuscript at www.biomedcentral.com/submit

) Biomed Central 